\title{
DETERMINACIÒN DE TIMOL Y CARVACROL EN HOJAS DE ORÉGANO POR HPLC FL
}

\author{
Lena Téllez", Fermín Arévalo ${ }^{a}$, Henry Juárez ${ }^{\mathrm{b}}$, Pedro Altamirano ${ }^{\mathrm{a}}$, Karina Ccapa ${ }^{\mathrm{a}}$, Jorge \\ Chávez ${ }^{c}$, Lizardo Visitación ${ }^{a}$
}

\begin{abstract}
RESUMEN
El orégano (Origanum vulgare L.) es una planta aromática originaria del mediterráneo; tradicionalmente es cultivada en la zona sur del Perú. Se adapta muy bien a los valles interandinos sobre los 2600 m.s.n.m. Debajo de esa altitud, la concentración de aceites esenciales (timol y carvacrol) disminuye. El desarrollo del presente método tiene por finalidad dotar de herramientas para tomar decisiones sobre la identificación de regiones donde uno o más ecotipos pueden desarrollarse potencialmente con respecto a la calidad de sus aceites esenciales en función al contenido de timol y carvacrol. Como parte de las actividades del proyecto fue necesario el desarrollo de un protocolo para la cuantificación por HPLC para el cultivo de orégano.

El método propuesto consiste de un sistema HPLC con detector de Fluorescencia, fase móvil isocrática de acetonitrilo y agua $(\mathrm{ACN}): \mathrm{H}_{2} \mathrm{O}(50: 50)$, columna Purospher ${ }^{\circledR}$ STAR rp-18e $(4,6$ x $150 \mathrm{~mm}, 5 \mu \mathrm{m}$ ), flujo de $1 \mathrm{~mL} / \mathrm{min}$, volumen de inyección de $20 \mu \mathrm{L}$ y un tiempo de corrida de 15 minutos. El método ha presentado una linealidad mayor a 0,999, precisión $\leq 2,27$ y 2,4 intra día, y $\leq 2,47$ y 1,94 inter diario para el carvacrol y timol, respectivamente. Los límites de detección determinado LOD y límite de cuantificación LOQ fue de 0,0007 - 0,002 mg/L para el timol y 0,002 - 0,005 mg/L para el carvacrol, con una recuperación de 98,68 \% y 90,95\%, respectivamente. Los resultados muestran que el protocolo desarrollado es un método adecuado para la determinación de timol y carvacrol en hojas de orégano.
\end{abstract}

Palabras clave: Orégano, timol, carvacrol, aceites esenciales, HPLC.

\section{DETERMINATION OF THYMOL AND CARVACROL ON OREGANO LEAVES VIA HPLC FL}

\begin{abstract}
Oregano (Origanum vulgare L.) is an aromatic native plant originated from the Mediterranean region and it's traditionally grown in the south of Peru. The crop has adapted well over 2600 m.a.s.l. Bellow this altitude, the concentration of essential oils (thymol and carvacrol) decreases. The development of this method is essential to provide tools for decision making to identify regions where one or more ecotypes can potentially grow and produce high quality of essential oils contents of thymol and carvacrol. As part of the activities, it was necessary to develop a protocol to quantify HPLC for the oregano crop.
\end{abstract}

a Centro de Investigación en Química, Toxicología y Biotecnología Ambiental del Departamento Académico de Química de la Facultad de Ciencias de la UNALM Lima-Perú.

ltellez@lamolina.edu.pe

Molinos Cusco SA, Kilometro 76, Cusipata, Quispicanchi, Cusco

Instituto de Investigación en Bioquímica y Biología Molecular de la UNALM Lima-Perú 
The proposed method uses a HPLC with a fluorescence detector, isocratic mobile phase of acetonitrile and water $(\mathrm{ACN}): \mathrm{H}_{2} \mathrm{O}$ (50:50), a column Purospher ${ }^{\circledR}$ STAR rp-18e (4.6 x $150 \mathrm{~mm}, 5 \mu \mathrm{m})$, flow $1 \mathrm{ml} /$ minute, injection volume of $20 \mu \mathrm{L}$ and run time of 15 minutes. The present method has a linearity greater than 0.999 , precision $\leq 2.27,2.4$ intra-day, and $\leq 2.47$ and 1.94 for daily measurements for carvacrol and thymol respectively. Detection limits (DL) and quantification limits (QL) were 0.0007 to $0.002 \mathrm{mg} / \mathrm{L}$ for, thymol and 0.002 to $0.005 \mathrm{mg} / \mathrm{L}$ for carvacrol with a recovery of $98.68 \%$ and $90.95 \%$ respectively. Results show that the protocol is an appropriate method to quantify carvacrol and thymol via HPLC in oregano leaves.

Key words: Oregano, Thymol, Carvacrol, essential oils, HPLC.

\section{INTRODUCCIÓN}

La determinación de timol y carvacrol en muestras de orégano y otras plantas, ha sido realizado por muchos autores utilizando métodos por HPLC ${ }^{1-5} \mathrm{y}$ cromatografía de gases con detector FID o de masas. ${ }^{1,3,67}$ La cromatografía de gases es muy útil para realizar el análisis cualitativo y cuantitativo de todos los componentes no volátiles del aceite esencial; pero no puede ser utilizado para dosis grandes. El método por HPLC es una técnica muy precisa para cuantificar los componentes volátiles y no volátiles de una planta ${ }^{1}$, logrando una buena separación y una rápida determinación del contenido de timol y carvacrol en el aceite esencial de orégano. La diferencia principal entre los diferentes métodos de determinación por HPLC radica en el tipo de detector a utilizar; éstos pueden ser: Detector por arreglo de diodos en el rango $\mathrm{UV}^{1,2,3,5}$ o de fluorescencia ${ }^{4}$. Los detectores de fluorescencia tienen la capacidad de medir señales de luz muy débiles comparados con los detectores de absorción UV tal como el DAD 8. Los diferentes métodos de determinación hacen uso de diferentes tipos de columnas de marcas registradas y diversas longitudes, pero todas tienen en común el uso de una columna de fase reversa C-18.

En el presente trabajo se validó el método de determinación por HPLC con detector de Fluoresencia de timol y carvacrol en muestras de Origanum vulgare, bajo las condiciones mencionadas en el artículo, utilizando la linealidad, límites de detección y cuantificación, selectividad, precisión, recuperación ${ }^{1,9,10}$. Para el caso de la selectividad se utilizó la resolución de picos cromatográficos ${ }^{11}$.

\section{PARTE EXPERIMENTAL}

La investigación se realizó en los Laboratorio del Departamento de Química de la Facultad de Ciencias de la UNALM, Laboratorio de Instrumentación del Instituto de Investigación en Bioquímica y Biología Molecular y los campos experimentales de cultivo de Molinos,Cusco S.A.

\section{Material de plantas}

Se colectó hojas de Origanum vulgare provenientes de zonas de cultivo en Tacna, Perú.

\section{Extracción del aceite esencial}

Se utilizó la hidrodestilación con trampa de Clevenger. Se tomó $1000 \mathrm{~g}$ de material fresco de hojas de $O$. vulgare. Se secó a temperatura ambiente en sombra, obteniéndose $200 \mathrm{~g}$ de hojas secas. Se extrajo durante 120 minutos. El aceite esencial obtenido fue secado con sulfato de sodio anhidro y almacenado $\mathrm{a}+4^{\circ} \mathrm{C}$ antes de su uso. 
Reactivos químicos

Agua grado HPLC.

Acetonitrilo grado HPLC.

\section{Instrumentación}

El equipo de HPLC utilizado es Hitachi Elite LaChromm, con el Software EZChrom Elite v3.3.2, equipado con bomba L-2130, válvula gradiente baja presión L-2130ACC, degasificadora L-2000ACC, Interface USB IFU, horno de columnas L-2350, Inyector manual 7725i-006, mezclador cuaternario de solventes; el detector utilizado fue el Hitachi FL Detector L-2485; se utilizó la longitud de onda de excitación: $230 \mathrm{~nm}$, longitud de onda de emisión: $280 \mathrm{~nm}$.

Los cromatogramas fueron elaborados en: Instituto de Investigación en Bioquímica y Biología Molecular de la UNALM, 2014.

\section{Cuantificación por Cromatografía Líquida de Alta Resolución}

\section{Acondicionamiento}

Se buscó las condiciones de estabilidad del sistema cromatográfico, previos a la corrida de los estándares o muestras. Con la finalidad de eliminar las impurezas de la muestra posterior a la corrida de la muestra, se realizó un lavado de la columna, de tal forma, que queda limpia para la inyección de la siguiente.

\section{Preparación de los estándares}

Las soluciones Stock de timol $(3 \mathrm{mg} / \mathrm{mL})$ y carvacrol $(0,3 \mathrm{mg} / \mathrm{mL})$ fueron preparadas separadamente con una mezcla de solventes $\mathrm{ACN}: \mathrm{H}_{2} \mathrm{O}(80: 20)$. Se preparó diferentes concentraciones a partir de la solución stock $(0,6-18 \mu \mathrm{g} / \mathrm{mL}$ de timol y $0,06-1,8 \mu \mathrm{g} / \mathrm{mL}$ para carvacrol); se plateó la curva de calibración.

\section{Preparación de la muestra}

Se utilizó $200 \mathrm{mg} / \mathrm{L}$ de muestra del aceite esencial disueltos en $\mathrm{ACN}: \mathrm{H}_{2} \mathrm{O}$ (80:20). Se inyectó por triplicado.

\section{Identificación y cuantificación}

Se determinó la identidad de los componentes de la muestra en base a los tiempos de retención de los estándares puros. Se determinó la concentración de los componentes de la muestra a partir de la curva de calibración por regresión lineal y luego se llevó a masa de la hoja.

\section{Validación}

\section{Linealidad}

Para la verificación de la distribución normal de los resultados, se evaluó la linealidad a través de la relación entre la concentración del timol - carvacrol y la absorbancia por el detector de FL-HPLC. La determinación del coeficiente $\left(\mathrm{r}^{2}\right)$ se calculó por ajuste de mínimos cuadrados. La línea de calibración fue réalizada a través de dos réplicas para concentración de timol o carvacrol, para conocer la extensión de la variabilidad total de la respuesta que puede ser explicada por el modelo de regresión lineal.

\section{Límites de detección y cuantificación}

Los límites de detección (LODs) y cuantificación (LOQs) fueron calculados usando las expresiones $3,3 \sigma / \mathrm{s}$ y $10 \sigma / \mathrm{s}$, respectivamente; en donde $\sigma$ es la desviación estándar interceptada y s es la pendiente de la curva de calibración. 


\section{Selectividad}

Para el desarrollo del método cromatográfico, se requiere una separación de los picos; se evaluó la resolución de los picos de timol y carvacrol.

\section{Precisión}

La precisión del método indica el grado de dispersión entre la determinación de la misma muestra. Tres muestras en tres intervalos $(80,100$ y $120 \%)$ fueron analizados en el mismo día (intra diario) y por tres días consecutivos (inter diario) y se determinó la desviación estándar relativa (\% RSD). Las tres muestras de cada nivel fueron preparadas e inyectadas en el HPLC por tres veces.

\section{Recuperación}

Este parámetro muestra la proximidad entre el valor experimental y el valor real. Esto asegura que no se ha producido pérdidas durante el proceso de análisis. Este método fue llevado por el método recuperación después de la adición de dos concentraciones de estándar 0,1 y 1,0 $\mathrm{ug} / \mathrm{mL}$ de carvacrol y 0,7 y $7,0 \mu \mathrm{g} / \mathrm{mL}$ de timol. Para cada nivel se realizó la determinación por triplicado en el equipo de HPLC. Se determinó con la muestras de menor concentración.

\section{RESULTADOS Y DISCUSIÓN}

\section{Condiciones cromatografícas}

Se realizó un acondicionamiento por 30 min con la mezcla acetonitrilo agua 50:50, flujo 1 $\mathrm{mL} / \mathrm{min}$, logrando la estabilización de la señal del equipo.

La corrida de los estándares fue realizada con una fase móvil isocrática formada por acetonitrilo y agua (ACN): $\mathrm{H}_{2} \mathrm{O}$ (50:50), columna Purospher ${ }^{\circledR}$ STAR rp-18e (4.6 x $150 \mathrm{~mm}, 5$ $\mu \mathrm{m})$, flujo de $1 \mathrm{~mL} / \mathrm{min}$., volumen de inyección de todas las muestras y estándares de $20 \mu \mathrm{L}$ y un tiempo de corrida de 15 minutos.

El lavado fue utilizando $5 \mathrm{~min} 100 \%$ agua, flujo $1 \mathrm{~mL} / \mathrm{min}, 5.1 \mathrm{~min} 50: 50 \%$ agua acetonitrilo flujo $1 \mathrm{~mL} / \mathrm{min}, 10 \mathrm{~min} 50: 50 \%$ agua acetonitrilo, flujo $2 \mathrm{~mL} / \mathrm{min}, 30$ - $60 \mathrm{~min}$ 50:50\% agua acetonitrilo flujo $1 \mathrm{~mL} / \mathrm{min}, 60.1$ a $90 \mathrm{~min} 50: 50 \%$ agua metanol, flujo $1 \mathrm{~mL} / \mathrm{min}$.

\section{Identificación}

Se determinó que los estándares de carvacrol y timol se encuentran bien definidos con tiempos de retención de 9,5 y 10,6 minutos, como se observa en el cromatograma siguiente (figura 1).

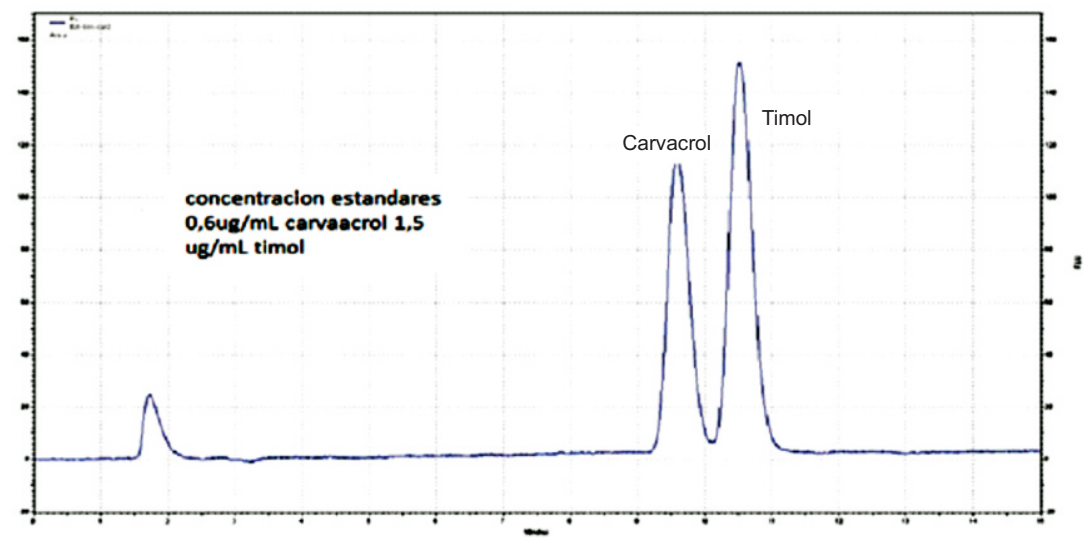

Figura 1. Cromatograma de los estándares de carvacrol y timol 


\section{Validación}

\section{Linealidad}

Como se observa en la figura 2 la curva de calibración del timol presenta un valor de $\mathrm{r}^{2} \mathrm{de}$ 0,999967 para concentraciones de 0,3 hasta $9,6 \mathrm{mg} / \mathrm{L}$

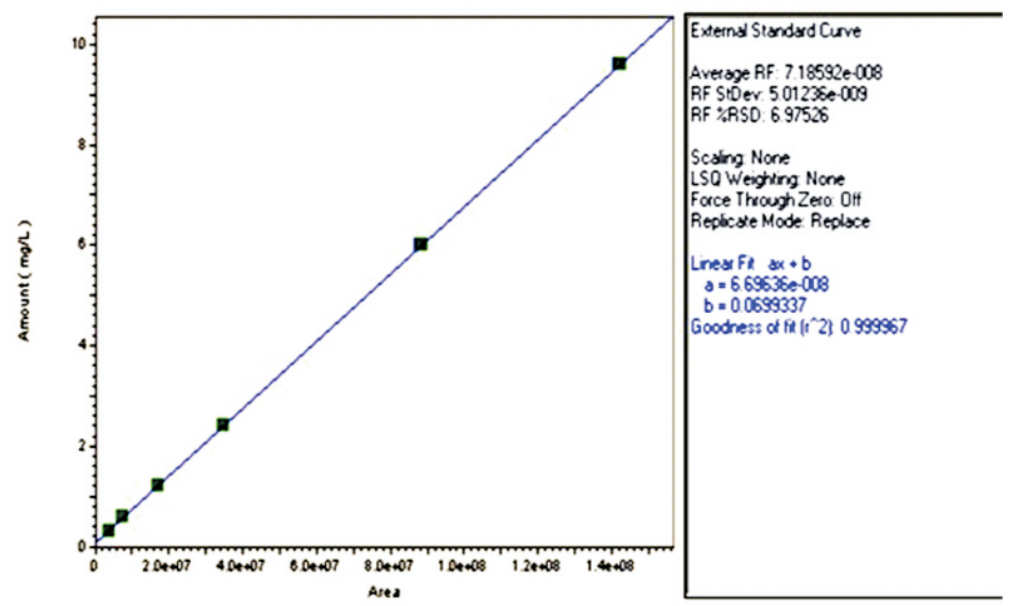

Figura 2. Curva de calibración del timol

En la figura 3 la curva de calibración del carvacrol presenta un valor de $r^{2}$ de 0,999811 para concentraciones de 0,03 hasta $0,96 \mathrm{mg} / \mathrm{L}$.

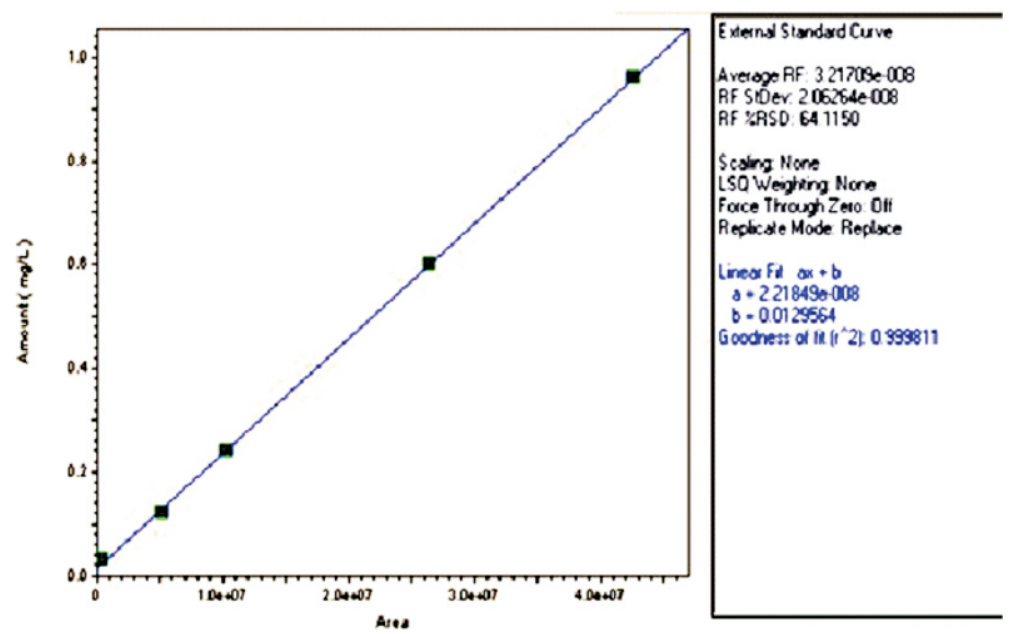

Figura 3. Curva de calibración del carvacrol 


\section{Límites de detección y cuantificación}

Para el timol, el límite de detección determinado (LOD) es $0,0007 \mathrm{mg} / \mathrm{L}$ y el límite de cuantificación (LOQ) es $0,002 \mathrm{mg} / \mathrm{L}$. Para el carvacrol, el límite de detección determinado LOD es $0,002 \mathrm{mg} / \mathrm{L}$ y el límite de cuantificación(LOQ) es $0,005 \mathrm{mg} / \mathrm{L}$.

\section{Selectividad}

Se ha determinado un valor de resolución Rs de 1,65, lo que indica una buena resolución de los picos de timol y carvacrol.

\section{Precisión}

La precisión de los resultados del análisis de timol y carvacrol, respectivamente, fue RSD $\% \leq$ 2,27 y 2,4 intra día, y RSD $\% \leq 2,47$ y 1,94 inter diario para el carvacrol y timol, respectivamente.

\section{Recuperación}

Se obtuvo una recuperación máxima de carvacrol y timol de 98,68 \% y 90,95 \% respectivamente para los dos niveles.

\section{Elección de la dilución óptima de las muestras}

De la muestra de $200 \mathrm{mg} / \mathrm{L}$ del aceite esencial disueltos en $\mathrm{ACN}: \mathrm{H}_{2} \mathrm{O}$ (80:20), se requirió una dilución 1:10. Se obtuvo el cromatograma de la figura 4, donde la concentración de carvacrol fue de $0,003 \mathrm{mg} / \mathrm{L}$ y la de timol fue de $0,189 \mathrm{mg} / \mathrm{L}$.

\section{Aplicación del método a muestras de orégano}

El método es de fácil aplicación para la muestra de orégano extraída por Klevenger.

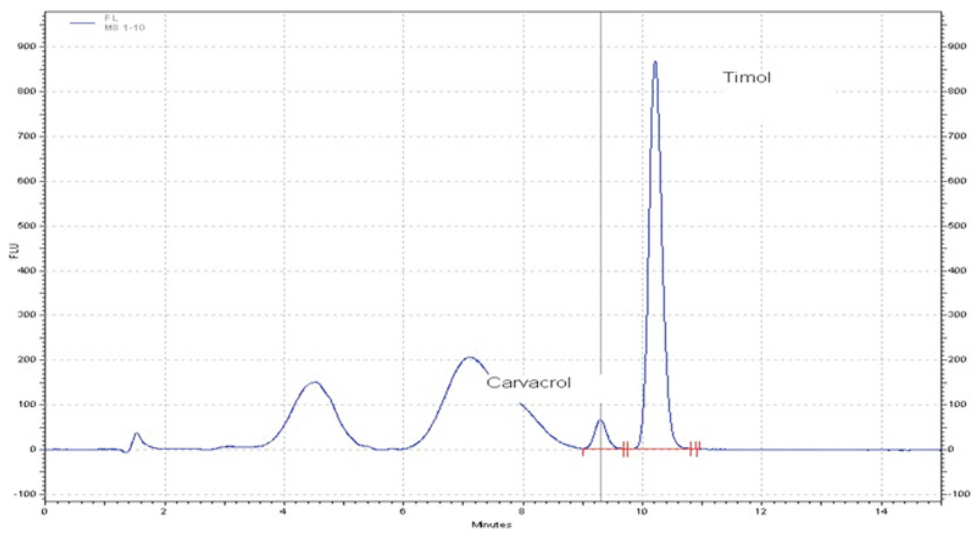

Figura 4. Muestra de Orégano.

\section{Expresión de resultados}

El porcentaje de carvacrol y timol en el aceite esencial:

$\mathrm{mg}$ carvacrol $/ 100 \mathrm{mg}$ aceite esencial $=L_{H P L C} * F * 100 / C o$

$\mathrm{mg}$ timol $/ 100 \mathrm{mg}$ aceite esencial $=L_{H P L C} * F * 100 / C o$

Donde:

$\mathrm{L}_{\text {HPLC }}$ : $\mathrm{mg} / \mathrm{L}$ de carvacrol o carvacrol medidos en el HPLC

F: $\quad$ Factor de dilución ejm: 1 en $10=10$

Co: Concentración del aceite esencial en la muestra a analizar mg/L 
La muestra analizada por este método contiene 1,04 y 19,95 \% de carvacrol y timol. Estos valores son similares a los encontrados para Origanum x aplii and Origanum x majoricum donde el timol presenta valores de 33,8 y $12,9 \%$, respectivamente y para el carvacrol se encontró valores menores a $1,0 \%{ }^{12}$.

\section{CONCLUSIONES}

El método de determinación es adecuado para la cuantificación del timol y carvacrol en muestras de hojas de orégano secadas al aire bajo sombre y extraídos por el método de Klevenger durante 2 horas.

\section{AGRADECIMIENTO}

El proyecto fue financiado por el Fondo de Innovación en Ciencia y Tecnología - FINCyT, Fondo de Investigación y Desarrollo Para la Competitividad (FIDECOM) con el Contrato PCyT - FIDECOM - Molinos Cusco - Convenio N049-2012. Este proyecto se desarrolló en conjunto entre Molinos Cusco SA y el Centro de Investigación en Química Toxicología y Biotecnología Ambiental (CIQTOBIA) del Departamento Académico de Química y el apoyo del Instituto de Investigación en Bioquímica y Biología Molecular de la UNALM.

\section{BIBLIOGRAFÍA}

1. H. Hajimehdipoor, M. Shekarchi, M. Khanavi, N. Abid, M. Amri, A validated high performance liquid chromatography method for the analysis of thymol and carvacrol in Thymus vulgaris L. volatile oil. Pharmacognosy Magazine; 2010; 6 (23): 154-158.

2. A. Ghasemi Pirbalouti1, M. Rahimmalek, F. Malekpoor, A. Karimi, Variation in antibacterial activity, thymol and carvacrol contents of wild populations of Thymus daenensis subsp. daenensis Celak. Plants Omics Journal; 2011; 4(4): 209-214.

3. I. S. Al-Sheibany, K. H. Kadeem, A. S. Abdullah. Isolation and Identification of Volatile oils from Iraqi Thyme ( Thymbra Spicata ) and study the antimicrobial activity. National Journal of Chemistry; 2005;18: 289-298

4. L. P. Roldán. Evaluación del uso de los aceites esenciales como alternativa al uso de antibióticos promotores de crecimiento en pollos de engorde. Tesis de Magíster en Producción Animal, línea de profundización en nutrición de monogástricos. Bogotá Colombia. Universidad Nacional de Colombia Facultad de Medicina Veterinaria y Zootécnia; $65-72,2010$.

5. V. Solinas, C. Gessa, L. Falchi Delitala, High-performance liquid chromatographic analysis of carvacrol and thymol in the essential oil of Thymus capitatus. Journal of Chromatography A; 1981;219, (2): 332-337.

6. C.C. Liolios, O. Gortzi, S. Lalas, J. Tsaknis, I. Chinou; Liposomal incorporation of carvacrol and thymol isolated from the essential oil of Origanum dictamnus L. and in vitro antimicrobial activity. Food Chemistry; 2009; 112, (1): 77-83.

7. D. R. Vásquez C. El orégano de monte (Lippia origanoides) del Alto Patía: Efecto del método de obtención de sus extractos sobre la composición y la actividad antioxidante de los mismos. Bogotá - Colombia. Tesis Maestría en Ciencias Química Universidad Nacional de Colombia; 29-30, 2012.

8. H. Franz, V. Jendreizik. Fluorescence Method Development Handbook. Thermo Fisher Scientific, Germering, Germany; 1 -2, 2013. 
9. Instituto de Salud Pública. Validación de métodos y determinación de la incertidumbre de la medición: "Aspectos generales sobre la validación de métodos". Santiago, Chile, 2145, 2010.

10. Methodology (CPMP/ICH/281/95) London: ICH Topic Q2B, Validation of Analytical Procedures. 6 November. 1996.

11. E.Katz, R.Eksteen, P. Schoenmakers, N. Miller. Hanbook of HPLC, Chromatographics science series, V 78: 66-67. 1998.

12. C. Amadio, M. E. Zimmermann, R. Medina, S. Miralles, C. Dediol. Aceite esencial de orégano: un potencial aditivo alimentario. Rev. FCA UNCUYO. ISSN 0370-4661. 2011; 43 (1): 237-245. 